PROCEEDINGS OF THE

AMERICAN MATHEMATICAL SOCIETY

Volume 126, Number 5, May 1998, Pages 1453-1459

S 0002-9939(98)04317-2

\title{
HAUSDORFF DIMENSION AND DOUBLING MEASURES ON METRIC SPACES
}

\author{
JANG-MEI WU
}

(Communicated by Albert Baernstein II)

\begin{abstract}
Vol'berg and Konyagin have proved that a compact metric space carries a nontrivial doubling measure if and only if it has finite uniform metric dimension. Their construction of doubling measures requires infinitely many adjustments. We give a simpler and more direct construction, and also prove that for any $\alpha>0$, the doubling measure may be chosen to have full measure on a set of Hausdorff dimension at most $\alpha$.
\end{abstract}

Let $(X, \rho)$ be a compact metric space. Vol'berg and Konyagin proved in [VK] that $(X, \rho)$ carries a nontrivial doubling measure $\mu$ (there exists $\Lambda \geq 1$ so that $\mu(B(x, 2 r)) \leq \Lambda \mu(B(x, r))$ for all $x \in X$ and $r>0)$ if and only if $(X, \rho)$ has finite uniform metric dimension (in each ball $B(x, 2 r)$, there exist at most $N$ points with mutual distances at least $r)$. Here $B(x, r)=\{y: \rho(x, y)<r\}$.

Assume that $(X, \rho)$ has finite uniform metric dimension. The construction of doubling measures in [VK] requires infinitely many adjustments which cannot be predicted in advance. In this note, we give a simpler and more direct construction, and prove that given any $\alpha>0$, there exists a doubling measure on $X$ that has full measure on a set of Hausdorff dimension at most $\alpha$. Also we observe that a doubling measure may be concentrated on a countable set even when $X$ is a set on the real line of positive length. Some ideas have been adapted from [FKP], [VK] and $[\mathrm{T}]$.

\section{Theorems AND EXAMPLES}

Assume, from now on, that $(X, \rho)$ is a compact metric space of finite uniform metric dimension and that diam $X<1$.

For each $k \geq 0$, let $S_{k}=\left\{x_{k, j}: 1 \leq j \leq J(k)\right\}$ be a maximal $10^{-k}$-net on $X$ (points in $S_{k}$ having mutual distances at least $10^{-k}$, and points outside $S_{k}$ having distances less than $10^{-k}$ to $S_{k}$ ), satisfying

$$
S_{0} \subseteq S_{1} \subseteq \cdots \subseteq S_{k} \subseteq S_{k+1} \subseteq \cdots .
$$

Note that $S_{0}$ has only one point $x_{0,1}$.

For each $k \geq 0$, let $\left\{T_{k, j}: 1 \leq j \leq J(k)\right\}$ be a partition of $S_{k+1}$ satisfying

$$
S_{k+1} \cap B\left(x_{k, j}, 10^{-k} / 2\right) \subseteq T_{k, j} \subseteq S_{k+1} \cap B\left(x_{k, j}, 10^{-k}\right) .
$$

Received by the editors October 24, 1996.

1991 Mathematics Subject Classification. Primary 28C15; Secondary 54E35, 54E45.

Key words and phrases. Doubling measure, metric space, Hausdorff dimension.

Partially supported by the National Science Foundation.

(C)1998 American Mathematical Society 
We call elements of $T_{k, j}$ branch points of $x_{k, j}$, the element $x_{k, j}$ an old branch point and the rest new branch points. Since $X$ has finite uniform metric dimension, $T_{k, j}$ has at most $N^{4}$ elements.

Let $M \geq N^{4}$, and let $w_{k, j}$ be weights at $x_{k, j}(k \geq 1)$ so that

$$
\begin{gathered}
M^{-1} \leq w_{k, j} \leq 1, \\
w_{k, j} \equiv w_{k} \quad \text { on } \quad S_{k} \backslash S_{k-1},
\end{gathered}
$$

and

$$
\sum_{x_{k+1, i} \in T_{k, j}} w_{k+1, i}=1
$$

Theorem 1. Assume that $\mu_{k}(k \geq 0)$ are measures on $X$ with total mass concentrated on $S_{k}$, defined as follows: $\mu_{0}$ is the unit point measure at $x_{0,1}$; after $\mu_{k}$ is chosen, $\mu_{k+1}$ is defined by distributing the mass from $x_{k, j}$ to its branch points in $T_{k, j}$ so that

$$
\mu_{k+1}\left(\left\{x_{k+1, i}\right\}\right)=w_{k+1, i} \mu_{k}\left(\left\{x_{k, j}\right\}\right), x_{k+1, i} \in T_{k, j} .
$$

Then $\left\{\mu_{k}\right\}$ converges in the weak star topology to a doubling measure $\mu$ on $(X, \rho)$ with

$$
\mu(B(x, 2 r)) \leq M^{3} N^{8} \mu(B(x, r))
$$

for each $x \in X$ and $r>0$.

This construction works because of (1.3) - the weight being a constant at all new branch points in any given generation. This allows us to compare measures of any two nearby branch points, regardless of their ancestors.

When $M$ is large, with a suitable choice of weights, the measure $\mu$ is concentrated on a small set. The next theorem extends a result of Tukia $[\mathrm{T}]$ on Euclidean space to metric spaces.

Theorem 2. Given $\alpha>0$, there exists a doubling measure on $(X, \rho)$ that has full measure on a set of Hausdorff dimension at most $\alpha$.

Recall that the $\beta$-dimensional Hausdorff content of a set $E$ in $X$ is the number $H_{\beta}(E)=\inf \sum_{j} r_{j}^{\beta}$, where the infimum is taken over all countable covers of $E$ by balls of radii $r_{j}$. The Hausdorff dimension of a set $E \operatorname{is~} \inf \left\{\beta: H_{\beta}(E)=0\right\}$.

A doubling measure on a ball in an Euclidean space cannot have full measure on a set of zero Hausdorff dimension. In contrast, the following examples exist for sets having no interiors.

Example 1. For each $\alpha \in[0,1]$, there exists a compact set $X \subseteq \mathbb{R}^{1}$ of Hausdorff dimension $\alpha$ so that every doubling measure on $X$ is purely atomic.

Example 2. There exists a compact set $X \subseteq \mathbb{R}^{1}$ of positive length, so that some doubling measures on $X$ are purely atomic.

Both examples are essentially in $[\mathrm{KW}]$ and were constructed for another purpose.

Let $E$ be the Cantor ternary set on the unit interval, $F$ be the midpoints of all complementary intervals and $X=E \cup F$. Then every doubling measure on $X$ is concentrated on $F$. A similar construction works for every $\alpha$ in $[0,1)$. When $\alpha=1$, we combine an appropriate sequence of such sets together with their limit points. 
As for Example 2, let $\nu$ be a doubling measure on $\mathbb{R}^{1}$ having full measure on a set of zero length as constructed in [BA], and let $E$ be a compact subset contained in $[0,1]$ having positive length and zero $\nu$-measure. Let $\mathcal{W}$ be a Whitney decomposition of $(-2,2) \backslash E$, and $F$ be the collection of midpoints of the intervals in $\mathcal{W}$. Let $X=E \cup F \cup\{-2,2\}$, and let $\mu$ be the measure on $X$ with total mass on $F$ so that at each $x \in F, \mu(\{x\})$ is the $\nu$-measure of the corresponding Whitney interval. Then $X$ and $\mu$ have the properties required.

For details, see the examples $X$ and $Z$ in $[\mathrm{KW}]$.

\section{Proof of Theorem 1}

Define history $h$ on $\bigcup_{k \geq 1} S_{k}$ as follows: $h(x)=\left(x_{0,1}, x\right)$ on $S_{1}$; and for $x \in T_{k, j} \subseteq$ $S_{k+1}, h(x)$ is the $(k+2)$-tuple $\left(a_{0}, a_{1}, \ldots, a_{k}, x\right)$, where $\left(a_{0}, a_{1}, \ldots, a_{k}\right)=h\left(x_{k, j}\right)$. We call $a_{m}(0 \leq m \leq k)$ the $m$-th generation ancestor of $x$. These are well-defined because $\left\{T_{k, j}: 1 \leq j \leq J(k)\right\}$ is a partition of $S_{k+1}$.

There is a slight abuse of notation: when $x_{k, j}$ and $x_{\ell, i}$ are the same point in $X$ while considered as branch points in two different generations, $h\left(x_{k, j}\right)$ and $h\left(x_{\ell, i}\right)$ have different numbers of components.

For $\ell \geq k+1$, let

$$
T_{k, j}^{\ell}=\left\{x \in S_{\ell}: \text { the } \ell \text { th generation ancestor of } x \text { is } x_{k, j}\right\},
$$

and call elements of $T_{k, j}^{\ell}$ the $\ell$ th generation branch points of $x_{k, j}$. Note that $T_{k, j}^{k+1}=$ $T_{k, j}$

$$
T_{k, j}^{\ell} \subseteq T_{k, j}^{\ell+1}
$$

and $\left\{T_{k, j}^{\ell}: 1 \leq j \leq J(k)\right\}$ is a partition of $S_{\ell}$. Denote by

$$
T_{k, j}^{\infty}=\bigcup_{\ell \geq k+1} T_{k, j}^{\ell}
$$

all branch points of $x_{k, j}$, and note that

$$
T_{k, j}^{\infty} \cap T_{m, i}^{\infty}=\emptyset
$$

if neither $x_{k, j}$ nor $x_{m, i}$ is an ancestor of the other.

We claim that for $\ell \geq k+1$,

$$
S_{\ell} \bigcap B\left(x_{k, j}, 10^{-k} / 3\right) \subseteq T_{k, j}^{\ell} \subseteq S_{\ell} \bigcap B\left(x_{k, j}, 10^{-k+1} / 9\right) ;
$$

thus

$$
\bigcup_{k+1}^{\infty} S_{\ell} \bigcap B\left(x_{k, j}, 10^{-k} / 3\right) \subseteq T_{k, j}^{\infty} \subseteq B\left(x_{k, j}, 10^{-k+1} / 9\right) .
$$

Therefore, any point in $\bigcup_{k+1}^{\infty} S_{\ell}$ which is sufficiently close to $x_{k, j}$ is a branch point of $x_{k, j}$, and all branch points of $x_{k, j}$ are not far from $x_{k, j}$. To prove (2.2) let $x \in T_{k, j}^{\ell}$ and follow along its ancestors since $x_{k, j}$; we have $\rho\left(x_{k, j}, x\right)<10^{-k}+$ $10^{-k-1}+\cdots+10^{-\ell+1}<10^{-k+1} / 9$; this proves the second inclusion in (2.2). If $x_{\ell, i} \in S_{\ell} \bigcap B\left(x_{k, j}, 10^{-k} / 3\right)$, then either $x_{\ell, i}=x_{k+1, p}$ or $x_{\ell, i} \in T_{k+1, p}^{\ell}$ for some $p$. Apply the second inclusion to $x_{k+1, p}$; we have $\rho\left(x_{\ell, i}, x_{k+1, p}\right)<10^{-k} / 9$, and hence $\rho\left(x_{k+1, p}, x_{k, j}\right)<10^{-k} / 9+10^{-k} / 3<10^{-k} / 2$. In view of (1.1), $x_{k+1, p} \in T_{k, j}$ and hence $x_{\ell, i} \in T_{k, j}^{\ell}$; this proves the first inclusion in (2.2). 
The convergence of $\left\{\mu_{k}\right\}$ is now clear.

We note from (1.3), (1.4), (1.5) and (2.1) that for $\ell \geq k+1$,

$$
\mu_{\ell}\left(T_{k, j}^{\ell}\right)=\mu_{k}\left(\left\{x_{k, j}\right\}\right),
$$

and

$$
\mu_{\ell}\left(\left\{x_{\ell, i}\right\}\right)=\left(\prod_{k+1}^{\ell} w_{m}\right) \mu_{k}\left(\left\{x_{k, j}\right\}\right),
$$

provided that $x_{\ell, i} \in T_{k, j}^{\ell}$, and $x_{\ell, i}$ and all ancestors since the $(k+1)$ st generation are new branch points.

The main idea of the proof is contained in the following lemma.

Lemma 1. If $k \geq 1$ and $\rho\left(x_{k, i}, x_{k, j}\right)<\frac{2}{9} 10^{-k+3}$, then

$$
\mu_{k}\left(\left\{x_{k, i}\right\}\right) / \mu_{k}\left(\left\{x_{k, j}\right\}\right) \leq M^{3} .
$$

Proof. For $k=1$, the estimate follows from (1.2) and (1.5). Assume $k \geq 2$ and let $h\left(x_{k, i}\right)=\left(a_{0}, a_{1}, \ldots, a_{k-1}, x_{k, i}\right), h\left(x_{k, j}\right)=\left(b_{0}, b_{1}, \ldots, b_{k-1}, x_{k, j}\right)$. Denote by $k_{0}$ the largest index for which $a_{k_{0}}=b_{k_{0}}$.

If $k_{0}<k-3$, we claim that $a_{m}$ and $b_{m}$ are new branch points in $S_{m}$ for each $m$ in $\left[k_{0}+2, k-2\right]$. Otherwise, assume that $a_{m}$ is an old branch point in $S_{m}$; thus $a_{m}$ and $a_{m-1}$ are the same point in $X$. Because $a_{m}$ is an ancestor of $x_{k, i}$, it follows from $(2.2)$ that $\rho\left(x_{k, i}, a_{m}\right)<10^{-m+1} / 9$. Because $a_{m-1} \neq b_{m-1}, a_{m-1}$ is not an ancestor of $x_{k, j}$; from (2.2) again, we have $\rho\left(x_{k, j}, a_{m-1}\right)>10^{-m+1} / 3$. Thus $\rho\left(x_{k, i}, x_{k, j}\right)>10^{-m+1} / 3-10^{-m+1} / 9>\frac{2}{9} 10^{-k+3}$, which is a contradiction. Therefore $a_{m}$, and similarly $b_{m}$, is a new branch point. In view of (2.4),

$$
\mu_{k-2}\left(\left\{a_{k-2}\right\}\right)=\left(\prod_{k_{0}+2}^{k-2} w_{\ell}\right) \mu_{k_{0}+1}\left(\left\{a_{k_{0}+1}\right\}\right)
$$

and

$$
\mu_{k-2}\left(\left\{b_{k-2}\right\}\right)=\left(\prod_{k_{0}+2}^{k-2} w_{\ell}\right) \mu_{k_{0}+1}\left(\left\{b_{k_{0}+1}\right\}\right) .
$$

As $a_{k_{0}+1}$ and $b_{k_{0}+1}$ are branch points of $a_{k_{0}}=b_{k_{0}}, \mu_{k_{0}+1}\left(\left\{a_{k_{0}+1}\right\}\right) / \mu_{k_{0}+1}\left(\left\{b_{k_{0}+1}\right\}\right)$ $\leq M$ by (1.2) and (1.5); similarly $M^{-2} \leq \mu_{k}\left(\left\{x_{k, i}\right\}\right) / \mu_{k-2}\left(\left\{a_{k-2}\right\}\right) \leq 1$ and $M^{-2}$ $\leq \mu_{k}\left(\left\{x_{k, j}\right\}\right) / \mu_{k-2}\left(\left\{b_{k-2}\right\}\right) \leq 1$. From these, $(2.5)$ follows.

If $k_{0} \geq k-3, \quad(2.5)$ holds because of (1.2) and (1.5).

Given $x \in X$ and $r>0$, we shall prove (1.6). Assume that $10^{-k}<r \leq 10^{-k+1}$ for some $k \geq 1$. Because $S_{k+1}$ is a maximal net, $\rho\left(x, x_{k+1, p}\right) \leq 10^{-k-1}$ for some $p$ and $T_{k+1, p}^{\infty} \subseteq B\left(x_{k+1, p}, 10^{-k} / 9\right) \subseteq B(x, r / 4)$. Therefore, by (2.3),

$$
\mu(B(x, r / 2)) \geq \mu\left(\overline{T_{k+1, p}^{\infty}}\right) \geq \mu_{k+1}\left(\left\{x_{k+1, p}\right\}\right) .
$$

Let $\mathcal{J}$ be the set of $j$ 's so that $x_{k+1, j} \in B(x, 2 r)$; then $\mathcal{J}$ contains at most $N^{8}$ elements. We claim that

$$
S_{\ell} \cap B(x, 3 r / 2) \subseteq \bigcup_{\mathcal{J}} T_{k+1, j}^{\ell} \quad \text { for each } \quad \ell \geq k+2 .
$$


In fact, given $x_{\ell, i} \in B(x, 3 r / 2), x_{\ell, i}$ is contained in $T_{k+1, q}^{\ell}$ for some $q$. Since $T_{k+1, q}^{\ell} \subseteq B\left(x_{k+1, q}, 10^{-k} / 9\right)$, we have $\rho\left(x_{k+1, q}, x\right) \leq \rho\left(x_{k+1, q}, x_{\ell, i}\right)+\rho\left(x_{\ell, i}, x\right)<$ $10^{-k} / 9+3 r / 2<2 r$. Thus $q \in \mathcal{J}$. This proves (2.7). Therefore

$$
\mu_{\ell}(B(x, 3 r / 2)) \leq \sum_{\mathcal{J}} \mu_{\ell}\left(T_{k+1, j}^{\ell}\right)=\sum_{\mathcal{J}} \mu_{k+1}\left(\left\{x_{k+1, j}\right\}\right)
$$

for each $\ell \geq k+2$. Since $\rho\left(x_{k+1, p}, x_{k+1, j}\right) \leq \rho\left(x_{k+1, p}, x\right)+\rho\left(x, x_{k+1, j}\right)<10^{-k-1}+$ $2 r<\frac{2}{9} 10^{-k+1}$, we deduce from (2.5) and (2.6) that

$$
\mu_{\ell}(B(x, 3 r / 2)) \leq M^{3} N^{8} \mu(B(x, r / 2) .
$$

From this, (1.6) follows. And this proves Theorem 1.

\section{Proof of Theorem 2}

For $x \in S_{k}$, recall that $h(x)$ has the form $\left(x_{0,1}, a_{1}, a_{2}, \ldots, a_{k-1}, a_{k}\right)$ and that the first element $x_{0,1}$ is not a branch point. For $k \geq 1$ and $0 \leq p \leq k$, denote by

$$
S_{k}(p)=\left\{x \in S_{k}: h(x) \quad \text { contains exactly } p \text { old branch points }\right\}
$$

There are exactly $\left(\begin{array}{l}k \\ p\end{array}\right)$ different ways to position $p$ old branch points in $h(x)$; afterwards there are at most $(N-1)^{k-p}$ different ways to place new branch points in the remaining slots. Therefore $S_{k}(p)$ has at most $\left(\begin{array}{l}k \\ p\end{array}\right)(N-1)^{k-p}$ elements. Thus the set

$$
\sigma_{k}(p)=\left\{x \in S_{k}: h(x) \text { contains at least } p \text { old branch points }\right\}
$$

has at most $\sum_{m=p}^{k}\left(\begin{array}{c}k \\ m\end{array}\right)(N-1)^{k-m}$ elements.

Denoting $\frac{N-1}{M}$ by $\gamma$, we prove the following.

Lemma 2. If $k \geq 1$, then

$$
\mu_{k}\left(\sigma_{k}(p)\right) \geq \sum_{m=p}^{k}\left(\begin{array}{l}
k \\
m
\end{array}\right)(1-\gamma)^{m} \gamma^{k-m} \quad \text { for } \quad 0 \leq p \leq k .
$$

Proof. If $k=1$ and $p=0$, then $\sigma_{1}(0)=S_{1}$ and $\mu_{1}\left(\sigma_{1}(0)\right)=1$. If $k=1$ and $p=1$, then $\sigma_{1}(1)=\left\{\right.$ the old branch point in $\left.S_{1}\right\}$ and $\mu_{1}\left(\sigma_{1}(1)\right) \geq 1-\gamma$. Hence (3.1) holds for $k=1$.

Assume that (3.1) is true for some $k \geq 1$. We shall prove the inequality for $k+1$ and all $p$ in $[0, k+1]$. If $p=0$, then $\mu_{k+1}\left(\sigma_{k+1}(0)\right)=1$. If $p=k+1$, then $\mu_{k+1}\left(\sigma_{k+1}(k+1)\right) \geq(1-\gamma)^{k+1}$.

Let $1 \leq p \leq k$. For $x \in \sigma_{k+1}(p)$, denote by $a_{1}(x)$ the first generation ancestor of $x$. Then either $a_{1}(x)$ is an old branch point and there are at least $p-1$ old branch points in the remaining $k$ slots in $h(x)$, or $a_{1}(x)$ is a new branch point and there are at least $p$ old branch points in the remaining $k$ slots. From the induction 
hypothesis, it follows that

$$
\begin{aligned}
\mu_{k+1}\left(\sigma_{k+1}(p)\right)= & \mu_{1}\left(\sigma_{1}(1)\right) \sum_{m=p-1}^{k}\left(\begin{array}{l}
k \\
m
\end{array}\right)(1-\gamma)^{m} \gamma^{k-m} \\
& \quad+\left(1-\mu_{1}\left(\sigma_{1}(1)\right)\right) \sum_{m=p}^{k}\left(\begin{array}{l}
k \\
m
\end{array}\right)(1-\gamma)^{m} \gamma^{k-m} \\
\geq & (1-\gamma) \sum_{m=p-1}^{k}\left(\begin{array}{l}
k \\
m
\end{array}\right)(1-\gamma)^{m} \gamma^{k-m}+\gamma \sum_{m=p}^{k}\left(\begin{array}{l}
k \\
m
\end{array}\right)(1-\gamma)^{m} \gamma^{k-m} \\
= & \sum_{n=p}^{k+1}\left(\begin{array}{c}
k+1 \\
n
\end{array}\right)(1-\gamma)^{n} \gamma^{k+1-n} .
\end{aligned}
$$

The inequality follows from the fact that $\lambda A+(1-\lambda) a \geq(1-\gamma) A+\gamma a$ provided that $\lambda \geq 1-\gamma$ and $A \geq a>0$. Therefore (3.1) holds for $k+1$. The lemma is proved.

Assume that $M$ is large enough so that $\gamma=\frac{N-1}{M}<\frac{1}{5}$ and

$$
(1-2 \gamma)^{-(1-2 \gamma)}(2 \gamma)^{-2 \gamma}(2 N)^{2 \gamma} 10^{-\alpha}<2^{-\alpha} .
$$

Choose $p$ to be $[(1-2 \gamma) k]$ in the remaining part of the proof, and let

$$
\tau_{k}=\bigcup\left\{T_{k, j}^{\infty}: x_{k, j} \in \sigma_{k}(p)\right\} .
$$

Then for large $k$,

$$
\begin{aligned}
H_{\alpha}\left(\bar{\tau}_{k}\right) & \leq \sum_{m=p}^{k}\left(\begin{array}{l}
k \\
m
\end{array}\right)(N-1)^{k-m}\left(10^{-k+1}\right)^{\alpha} \\
& \leq 10 k\left(\begin{array}{l}
k \\
p
\end{array}\right) N^{k-p} 10^{-k \alpha} \\
& \leq(1-2 \gamma)^{-(1-2 \gamma) k-1 / 2}(2 \gamma)^{-2 \gamma k-1 / 2}(2 N)^{2 \gamma k} 10^{-\alpha k} \\
& <2^{-\alpha k} .
\end{aligned}
$$

The third inequality follows from Stirling's formula $\left(k ! \approx k^{k+1 / 2} e^{-k} \sqrt{2 \pi}\right)$. Note from (3.1) that, for large $k$,

$$
\begin{aligned}
\mu\left(\bar{\tau}_{k}\right) & \geq \mu_{k}\left(\sigma_{k}(p)\right) \\
& =\sum_{m=p}^{k}\left(\begin{array}{c}
k \\
m
\end{array}\right)(1-\gamma)^{m} \gamma^{k-m} \\
& =1-\sum_{m=0}^{p-1}\left(\begin{array}{l}
k \\
m
\end{array}\right)(1-\gamma)^{m} \gamma^{k-m} \\
& >1-p\left(\begin{array}{l}
k \\
p
\end{array}\right)(1-\gamma)^{p} \gamma^{k-p} \\
& >1-10\left(\frac{e}{4}\right)^{\gamma k} .
\end{aligned}
$$

Here Stirling's formula is again used in the last estimate. 
Let

$$
\tau=\bigcap_{K \geq 5} \bigcup_{k \geq K} \bar{\tau}_{k}
$$

It follows from (3.2) and (3.3) that

$$
H_{\alpha}(\tau)=0 \quad \text { and } \quad \mu(\tau)=1 .
$$

This proves Theorem 2 .

\section{REFERENCES}

[BA] A. Beurling and L. Ahlfors, The boundary correspondence under quasiconformal mapping, Acta Math. 96 (1956), 125-142. MR 19:258c

[FKP] R. Fefferman, C. Kenig and J. Pipher, The theory of weights and the Dirichlet problem for elliptic equations, Ann. of Math. 134 (1991), 65-124. MR 93h:31010

[KW] R. Kaufman and J.-M. Wu, Two problems on doubling measures, Revista Mat. Iberoamericana 11 (1995), 527-545. CMP 96:05

[T] P. Tukia, Hausdorff dimension and quasisymmetric mappings, Math. Scand. 65 (1989), 152-160. MR 92b:30026

[VK] A.L. Vol'berg and S.V. Konyagin, On measures with the doubling condition, Math. USSR Izvestiya 30 (1988), 629-638. MR 88i:28006

Department of Mathematics, University of Illinois, Urbana, Illinois 61801 